\title{
Rotating Space Elevators: A New Venue in Space Elevator Physics
}

\author{
Leonardo Golubovic ${ }^{1} \&$ Steven Knudsen ${ }^{1}$ \\ ${ }^{1}$ Physics and Astronomy Department, West Virginia University, Morgantown, WV 26506-6515, USA \\ Correspondence: Leonardo Golubovic, Physics and Astronomy Department, West Virginia University, POB 6315, \\ Morgantown, WV 26506, USA. E-mail: lgolubov@wvu.edu
}

Received: November 7, 2016

Accepted: November 17, 2016 Online Published: December 31, 2016

doi:10.5539/apr.v9n1p56

URL: http://dx.doi.org/10.5539/apr.v9n1p56

\begin{abstract}
The physics of Space Elevators connecting the Earth with outer space has recently attracted increased attention, in part due to the discovery of ultra-strong materials such as carbon nanotubes and diamond nano-thread structures. In this article we review a new venue in space elevator physics: Rotating Space Elevators (RSE) [Golubović, L. \& Knudsen, S. (2009). Classical and statistical mechanics of celestial scale spinning strings: Rotating space elevators. Europhysics Letters 86(3), 34001.]. The RSE is a double rotating system of strings reaching outer space. Objects sliding along the RSE string (sliding climbers) do not require internal engines or propulsion to be transported far away from the Earth's surface. The RSE thus solves a major problem in the space elevator technology which is how to supply the energy to the climbers moving along the string. RSE strings exhibit interesting nonlinear dynamics and statistical physics phenomena. Satellites and spacecraft carried by sliding climbers can be released (launched) along RSEs. RSE strings can host space stations and research posts. Sliding climbers can be then used to transport useful loads and humans from the Earth to these outer space locations.
\end{abstract}

Keywords: Space Elevator, Inertial Forces, Classical Mechanics, Statistical Physics, Space Travel, Nonlinear Dynamics, Instabilities and Transitions, Chaos

\section{Introduction}

The physics of Space Elevators connecting the Earth with outer space has attracted increased attention in this millennium (Edwards \& Westling, 2003). This interest emerged in part due to the discovery of ultra-strong materials such as carbon nanotubes (Yu et al., 2000a, Yu et al., 2000b) and diamond nano-thread structures (Fitzgibbons et al., 2014). Space elevators are celestial scale examples of physical systems with reduced dimensionality such as the strings, polymers, and membranes (Kardar, 2007; Nelson, 2002; Nelson, Piran \& Weinberg, 1988). The classical and statistical mechanics of space elevators represents a bold extension of previous studies of satellite dynamics such as those of Beletskii (1965) and Hughes (2012).

Recently, a new concept has emerged in this applied physics area: Rotating Space Elevator (RSE) (Golubovic \& Knudsen, 2009; Knudsen \& Golubovic, 2014; Knudsen \& Golubovic, 2015). In this review article we discuss this new venue in space elevator physics. The RSE is a double rotating floppy string reaching extraterrestrial locations. Interestingly, objects sliding along the RSE string (climbers) do not require internal engines or propulsion to be transported far away from the Earth's surface. The RSE thus solves a major problem in space elevator physics which is how to supply energy to the climbers moving along space elevator strings.

\section{Historic Background: The Conceptual Development of the Traditional Space Elevator}

Dreams of traveling to the heavens have entranced men since the early times of civilization. The story of the "Tower of Babel" in Genesis 11 of the Bible connects the notion of human cooperation for space travel to "heaven" to the multiplying of human languages, which frustrates the effort. In modern history, the fable "Jack and the Beanstalk," from 1807 (and a burlesque version named The Story of Jack Spriggins and the Enchanted Bean from 1734) presents a young boy whose mother plants foolishly obtained seeds which then grow into a great tower that can even hold a giant! Neither of these stories addresses the physics questions of how the towers can remain upright under compressive and buckling (bending) forces.

It was therefore up to the famous Russian scientist Konstantin Tsiolkovsky in 1895 to integrate the vision of the space elevator with the realities of physics (Edwards \& Westling, 2003). Tsiolkovsky was considered to be a rocket scientist and the father of spaceflight and he had spent considerable time thinking about the limitations and alternatives of rocket flight. He was inspired by the Eiffel Tower in Paris to conceptualize a tower that 
reached from ground zero all the way into deep space, above the geosynchronous satellite orbit. This "celestial castle" would orbit the Earth in a geosynchronous fashion meaning that it would be directly overhead one spot on Earth's surface at all times. An object released at the tower's top would also have the orbital velocity necessary to remain in geosynchronous orbit. Thus, the Tsiolkovsky's tower can be used to deploy satellites into orbits around the Earth.

The centrifugal force due to Earth rotation acting on celestial towers has an interesting effect: A tall enough tower is under tension rather than compression, and therefore is not subject to the sorts of buckling that limits the height of skyscrapers. In the case of skyscrapers, the centrifugal force is negligible, but for the celestial size objects envisioned by Tsiolkovsky and his followers, both scientists and science fiction writers such as Arthur C. Clarke $(1978,1982)$, the gravitational force and centrifugal force play equally significant roles. Because the internal force is a tension rather than compression, the space elevator can be a floppy non-rigid object ("string").

It wasn't until 1960 that someone suggested a feasible method for building the space elevator. Another Russian scientist, Yuri N. Artsutanov, conceived a scheme for building a space tower (Artsutanov, 1960, July 31; Lvov, 1967). Artsutanov proposed using a geosynchronous satellite as the starting point from which to construct the tower. By using a counterweight, a cable would be lowered from the satellite down to the Earth surface while the counterweight was extended from the satellite away from Earth, keeping the center of gravity of the cable motionless relative to the rotating Earth. This construction scheme is still the standard (Edwards \& Westling, 2003).

Jerome Pearson (1975) brought the idea of the space elevator to the scientific community in the U.S. In his careful and detailed design of a workable space elevator while at the U.S. Air Force Flight Dynamics Laboratory he outlined, mathematically and physically, the implications of a space elevator string designed to have the constraint of constant stress (tension/cross sectional area) throughout, while maintaining an external force balance. Artsutanov independently proposed the same idea (Artsutanov, 1960, July 31; Lvov, 1967). The two balancing external forces in the earth frame are the centrifugal and gravitational forces. The Pearson-Artsutanov constant stress elevator provides a simple way to handle the high tensions present in space elevators: The elevator can be designed for any given value of the constant stress. This value is can be chosen to be smaller than the critical breaking stress of the material used. Hence, from the materials science point of view, real space elevators can be made. This spurred a lot of recent interest in building space elevators out of novel materials such as carbon nanotubes (Yu et al., 2000a, Yu et al., 2000b, Edwards \& Westling, 2003).

It is very easy to understand the advantages of the space elevator concept over conventional rocket propulsion. With chemical propulsion, a rocket carries its own fuel that it needs to overcome gravitational forces, leading to intrinsic energy inefficiency. Because of earth's deep gravitational well, the load-to-fuel ratios are typically very small (e.g., $\sim 10^{-2}$ for the Apollo/Saturn V missions to the Moon), so that essentially all fuel energy is used to accelerate the fuel itself. On the other side, within the space elevator concept, a spaceship climbs along the elevator via an internal electrical engine which uses externally supplied electric energy. Since there is no fuel carried by the climber, the supplied energy is $100 \%$ used to lift the climber. So, the space elevator concept is immensely more energy efficient than the rocket propulsion.

The problem however remains on how to externally supply the energy to the climber. Naively, one may think of running an electrical transmission line along the space elevator, until realizing just how long this structure is compared to transmission lines on earth, so that power losses will be close to $100 \%$. To remedy this, Edwards proposes that laser power be beamed up the elevator from the ground to the climber (Edwards \& Westling (2003)). The beam energy would be absorbed by climbers and converted into electrical energy driving their engines. For any of these schemes climbing is typically slow and it may take several months for the climber to travel along the space elevator from the Earth to the geosynchronous level. During such a long climb, the useful load (including possibly humans) would be exposed for a very long time to dangerous cosmic radiation, which is especially strong in this range of altitudes above the Earth. Even without this problem with cosmic rays, typically long travel time itself is certainly not a satisfactory feature of space elevators, especially if a rapid deployment of objects into outer space is desired.

\section{Rotating Space Elevator (RSE): Solution of the Climbers Energy Supply Problem}

In this review article we discuss a novel class of nonlinear dynamical systems, Rotating Space Elevators (RSE). The RSE concept has been introduced for the first time by Golubovic and Knudsen (2009) and elaborated in detail in their subsequent studies (Knudsen \& Golubovic, 2014; Knudsen \& Golubovic, 2015). 
a)

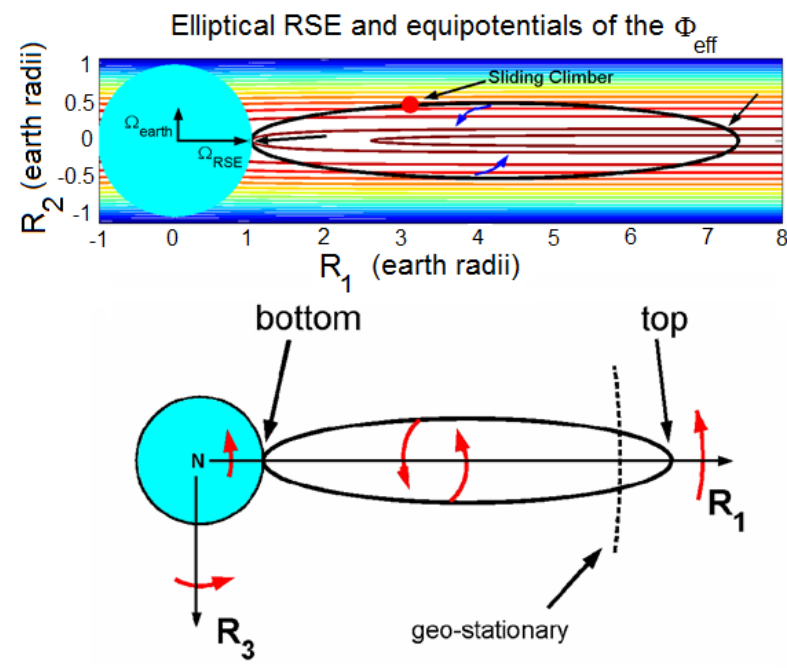

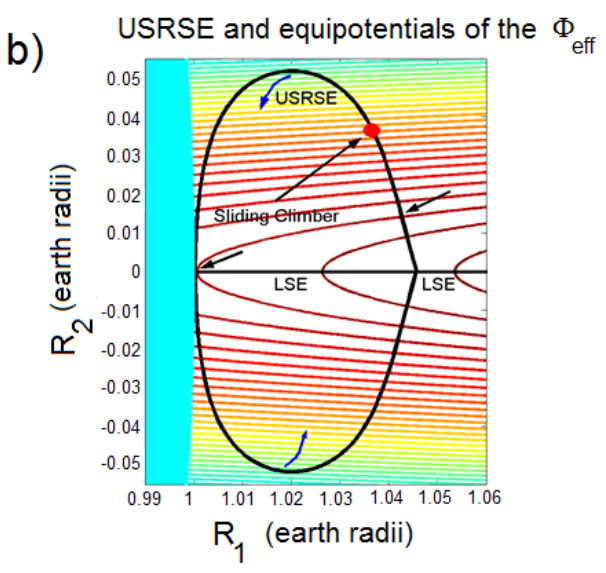

Figure 1. In (a) upper panel, the elliptical RSE with minor semi-axis $b=0.5$ Earth radii and major semi-axis $a=$ 3.2107 Earth radii (so its top is about 0.8 Earth radii above the geo-stationary level). In (a) lower panel, a different view on the RSE. The coordinate system $\left(R_{1}, R_{2}, R_{3}\right)$ rotates together with the Earth around the

$R_{2}$-axis pointing through the north pole $\mathrm{N}$. Indicated are the internal (nearly around the $R_{1}$-axis) and geo-synchronous (together with the Earth) rotations of the RSE. The RSE is tied to the Earth at its bottom point. In (b), we show the USRSE (attached to a LSE) with $T_{R S E}=4.22 \mathrm{~min}$ (discussed in sect. 4). In these figures we also include the equipotentials of the effective potential in eq. (7). Sliding climbers oscillate between two turning points (indicated by straight arrows) that are on the same equipotential. Adapted from Golubovic and Knudsen

The RSEs are multiply rotating systems of strings. Remarkably, useful loads and humans sliding along the RSE strings do not require internal engines or propulsion to be rapidly transported (sled) into space far away from the Earth's surface; see section 4 discussions. Thus, the RSE concept solves the major problem of energy supply to climbers that troubles the ordinary LSE concept.

The RSE is a double rotating floppy string typically having the shape of a loop as in Figure 1. Due to its special kind of motion (see below), the RSE becomes pre-tensioned due to gravitational and inertial forces. Due to the tension, the floppy RSE maintains its loopy shape.

The special RSE motion, ensuring the persistence of its shape, is a nearly a geometrical superposition of: (a) geosynchronous (one day period) rotation around the Earth, i.e. the $\mathrm{R}_{2}$-axis in Figure 1, and (b) yet another rotational motion of the string which is typically much faster (with period $\sim$ tens of minutes) and goes on around a line perpendicular to the Earth at its equator (the $\mathrm{R}_{1}$ axis in Figure 1).

This second, internal rotation plays a very special role: It provides the dynamical stability of the RSE shape and, importantly, it also provides a mechanism for the climbing of objects free to slide along the RSE string.

RSE can be used to elevate climbers from the surface of the Earth to remote outer space locations in a simple way; see Sec. 4. Remarkably, the climbers in Figure 1 do not need any internal engine to execute their motion. Rather, they spontaneously slide along the RSE string from the Earth to outer space locations. This unusual climber sliding motion is facilitated by the inertial force (centrifugal force) acting on climbers due to the RSE's internal rotation. In section 6 we describe possible use of RSE to launch satellites and interplanetary spaceships.

The RSE can be made in various shapes. The simple double rotating geometrical motion can be made to represent an approximate yet exceedingly accurate solution to the exact equations of the RSE string dynamics. This RSE feature is accomplished by a special ('magical') choice of mass distribution of the RSE cable; see Sec. 4. This RSE feature is corroborated by numerical simulations showing that (under the conditions discussed in Sec. 5) the RSE double rotation motion as well as nearly constant RSE shape can both persist indefinitely in time (Golubovic \& Knudsen, 2009; Knudsen \& Golubovic, 2014; Knudsen \& Golubovic, 2015). 
The elliptical RSE in Figure 1(a) exhibits very high tensile stresses at its points near mid-height. Therefore, other shapes were described whose mass distribution yields a Uniform Stress RSE (USRSE) (Golubovic \& Knudsen, 2009, Knudsen \& Golubovic, 2014). The USRSE, displayed in Figure 1(b), can be made by using technologically available materials such as carbon nanotubes; see section 4 .

\section{The physics of RSE}

In this section we review the basic physics of RSE (Golubovic \& Knudsen, 2009). For simplicity, let us consider inextensible limit in which the RSE floppy string obeys the Newtonian equation of motion

$$
\mu(s) \frac{\partial^{2} \vec{R}}{\partial t^{2}}=\frac{\partial}{\partial s} T(s, t) \frac{\partial}{\partial s} \vec{R}+\vec{f}_{e x t}
$$

In Eq. (1), $\vec{R}(s, t)$ are 3-D space positions of string points parametrized by their arc-length distances $s$ [thus, $|\partial \vec{R}(s, t) / \partial s|=1] . \mu(s)$ in Equation (1) is the string's local mass line density, while $T(s, t)$ is the local value of the string's tension field, and $\vec{f}_{\text {ext }}$ are external forces (per unit length) acting on the string. Prominent among them is the force of the Earth gravity, for which,

$$
\vec{f}_{\text {ext }}^{\text {grav }}=\mu(s) \vec{a}_{\text {grav }},
$$

with

$$
\vec{a}_{\text {grav }}=-\frac{\partial \Phi_{\text {grav }}}{\partial \vec{R}}
$$

Here,

$$
\Phi_{\text {grav }}(\vec{R})=-\frac{G M_{\text {earth }}}{|\vec{R}|}
$$

is the Earth's gravitational potential with $M_{\text {earth }}=$ the Earth's mass. $T(s, t)$ is obtained by solving Eq. (1) combined with the local constraint $|\hat{t}(s, t)|=1$, with $\hat{t}(s, t)=\partial \vec{R} / \partial s$. In the presence of a sliding climber of mass $m_{c l}$ at the arc-length distance $s(t)$, i.e., at the 3-D position $\vec{R}^{c l}(t)=\vec{R}(s(t), t)$, the external force density acting on the RSE, $\vec{f}_{\text {ext }}$ in Eq. (1) includes also the term

$$
\vec{f}_{\text {ext }}^{c l}=-\delta(s-s(t)) \vec{N},
$$

where $\vec{N}$ is the normal force exerted by the climber on the string; $\vec{N} \cdot \hat{t}=0$. The climber dynamics is governed by the second Newton's law,

$$
m_{c l} \frac{d^{2} \vec{R}^{c l}}{d t^{2}}=\vec{N}+\vec{F}_{e x t}
$$

with $\vec{F}_{\text {ext }}$ signifying (other than $\vec{N}$ ) external forces acting on the climber, e.g., the Earth's gravity,

$$
\vec{F}_{\text {ext }}^{\text {grav }}=m_{c l} \vec{a}_{\text {grav }}=-m_{c l} \frac{\partial \Phi_{\text {grav }}}{\partial \vec{R}} .
$$

Using the constraint $|\hat{t}(s, t)|=1$, from the above equations one finds

$$
\ddot{s}(t)=\hat{t} \cdot\left(\frac{\vec{F}_{e x t}}{m_{c l}}-\frac{\partial^{2} \vec{R}}{\partial t^{2}}\right),
$$

and

$$
\vec{N}=-\left(\vec{F}_{e x t}\right)_{\perp}+m_{c l}\left(\frac{\partial^{2} \vec{R}}{\partial t^{2}}\right)_{\perp}+m_{c l} \frac{\partial \hat{t}}{\partial s}[\dot{s}(t)]^{2}+2 m_{c l} \frac{\partial \hat{t}}{\partial t} \dot{s}(t)
$$


with $(\vec{V})_{\perp}=\vec{V}-\hat{t}(\hat{t} \cdot \vec{V})$ for any vector $\vec{V}$.

Space elevators such as RSE are most naturally discussed by using non-inertial reference frames. In a non-inertial frame rotating with the angular velocity $\vec{\Omega}$, inertial forces have to be included into $\vec{f}_{\text {ext }}$ and $\vec{F}_{\text {ext }}$ in Eqs. (1) and (1'), yielding

$$
\vec{f}_{\text {ext }}=\mu(s)\left(\vec{a}_{\text {grav }}+\vec{a}_{\text {inert }}\right), \vec{F}_{\text {ext }}=m_{c l}\left(\vec{a}_{\text {grav }}+\vec{a}_{\text {inert }}\right),
$$

with,

$$
\vec{a}_{\text {inert }}=\vec{\Omega} \times(\vec{R} \times \vec{\Omega})+\vec{R} \times \frac{d \vec{\Omega}}{d t}+\frac{d \vec{R}}{d t} \times 2 \vec{\Omega},
$$

(Landau \& Lifshitz, 1976). In the geosynchronous frame (used in the simulations and the figures displayed here) rotating with the period $=2 \pi / \Omega_{\text {earth }}=$ one day, Eq. (3) is employed with

$$
\vec{\Omega}=\vec{\Omega}_{\text {earth }}=\hat{e}_{2} \Omega_{\text {earth }},
$$

with unit vector $\hat{e}_{2}$ along the Earth polar axis and the equator in the $\left(R_{1}, R_{3}\right)$ plane; see Figs. 1(a) and (b). In the simulations, at $t=0$ the RSE is initially in the $\left(R_{1}, R_{2}\right)$ plane. To initiate the double rotation motion, the RSE is given initial spin around the $R_{1}$-axis, with the angular velocity $\Omega_{R S E}$. The RSE bottom point is tied to the Earth to provide access for the sliding climbers starting there their trip into outer space. Other than this, the RSE moves purely under the influence of inertia and gravity.

A remarkable effect of the RSE double rotation motion is that it facilitates a physical mechanism which efficiently moves sliding engine free climbers from the surface of the Earth to remote extraterrestrial locations. As evidenced by the simulations in Figure 2, a sliding climber starting at rest close to the Earth spontaneously oscillates between its initial position and a turning point in outer space. The nearly periodic character of the climber's motion is explained later on in this section.
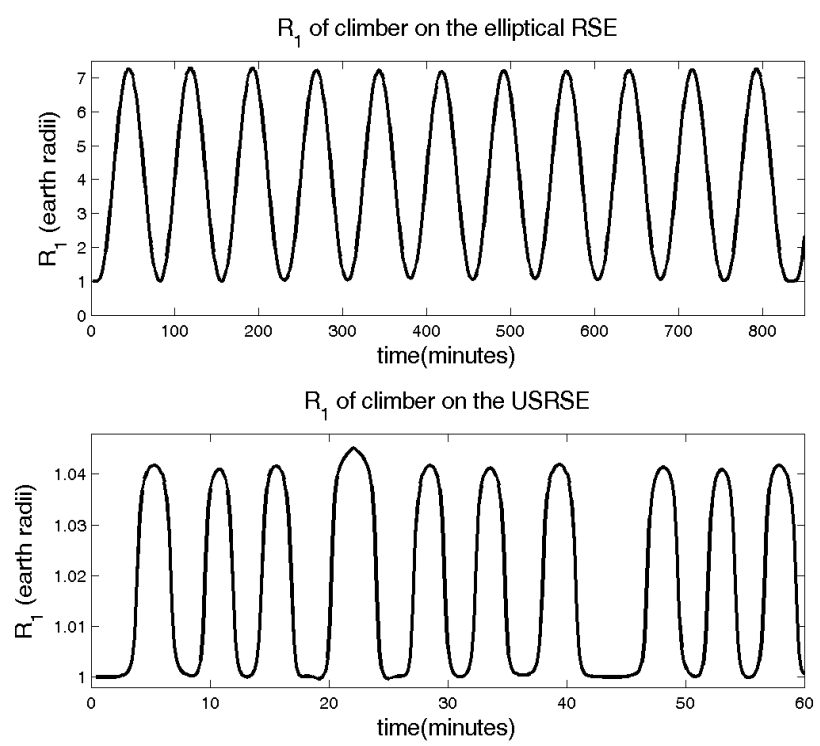

Figure 2. From the simulations of Knudsen and Golubovic (2014), the upper panel: The $R_{1}(t)$ coordinate of the climber which slides with no friction along the floppy RSE with the (initial) shape in Figure 1(a) and

$T_{R S E}=10.83 \mathrm{~min}$. The lower panel: The $R_{1}(t)$ coordinate of the climber on the floppy RSE with initial shape in Figure 1(b) with $T_{R S E}=4.22 \mathrm{~min}$. See Sec. 4 for the analytic explanation of the nearly periodic character of climber motion. Note: With a weak sliding friction, climbers would eventually stop near the RSE point minimizing the $U(s)=\Phi_{\text {eff }}(\vec{R}(s))$. From the equipotentials of the effective potential labeled in Figure 1, one can see that this point occurs close to the RSE point maximizing its $R_{2}$ coordinate in Figure 1 
Another remarkable effect is an enduring stability of the RSE sizes and orientation and the persistence the RSE's double rotation motion which is provided by a specially chosen form of the mass line density $\mu(s)$; see Eq. (8) below and Figure 3. This effect is documented by the simulations results displayed in Figure 4 (Golubovic $\&$ Knudsen, 2009; Knudsen \& Golubovic, 2014).

These two outstanding RSE effects are revealed by considering the system in the (natural for the RSE) double rotating frame (DRF) obtained from the geosynchronous (single rotating) frame by adding to it the rotation around the $R_{1}$-axis in Figs. 1(a) and (b). The net angular velocity of the DRF is thus $\vec{\Omega}(t)=\vec{\Omega}_{R S E}+\vec{\Omega}$ earth $(t)$. Here, $\vec{\Omega}_{R S E}=\Omega_{R S E} \hat{e}_{1}$ corresponds to the rotation around the $R_{1}$-axis while $\vec{\Omega}_{\text {earth }}(t)$ is the Earth's angular velocity vector which in the DRF rotates with the angular velocity $-\Omega_{R S E}$ (and thus acquires a time-dependence). With this $\vec{\Omega}(t)$, in DRF the Eq. (3) yields

$$
\vec{a}_{\text {inert }}=-\frac{\partial \Phi_{\text {inert }}}{\partial \vec{R}}+\vec{a}_{\text {res }},
$$

with

$$
\Phi_{\text {inert }}(\vec{R})=-\frac{1}{2} \Omega_{\text {earth }}^{2} R_{1}^{2}-\frac{1}{2}\left(\Omega_{R S E}^{2}+\frac{1}{2} \Omega_{\text {earth }}^{2}\right)\left(R_{2}^{2}+R_{3}^{2}\right),
$$

being a time-independent effective potential generating inertial forces sensed in the DRF. The residual, $\vec{a}_{\text {res }}$ term in Eq. (4) includes velocity dependent terms that vanish for an object at rest in the DRF, as well as fast time-dependent oscillatory terms of $\vec{a}_{\text {inert }}$ (with frequencies $\Omega_{R S E}$ and $2 \Omega_{R S E}>\Omega_{\text {earth }}$ ) that have zero time average over one RSE period $\left(T_{R S E}=2 \pi / \Omega_{R S E}\right)$; see Knudsen and Golubovic (2014). For the here interesting situations with $\Omega_{R S E}>\Omega_{\text {earth }}\left[T_{R S E} \sim 10 \mathrm{~min}<<T_{\text {earth }}=2 \pi / \Omega_{\text {earth }}=1\right.$ day $]$, the potential term in Eq. (4) dominates over the $\vec{a}_{r e s}$ term. Thus, Eq. (2) reduces to

$$
\vec{f}_{e x t} \cong-\mu(s) \frac{\partial \Phi_{e f f}}{\partial \vec{R}}, \quad \vec{F}_{e x t} \cong-m_{c l} \frac{\partial \Phi_{e f f}}{\partial \vec{R}},
$$

with the net effective potential,

$$
\Phi_{\text {eff }}(\vec{R})=\Phi_{\text {grav }}(\vec{R})+\Phi_{\text {inert }}(\vec{R})=-G \frac{M_{\text {earth }}}{|\vec{R}|}+\Phi_{\text {inert }}(\vec{R}) .
$$

The time independence of the $\Phi_{\text {eff }}(\vec{R})$ allowed Golubovic and Knudsen (2009) to find a special ('magical') mass distribution $\mu(s)$ with which the RSE string indefinitely maintains its initial shape by remaining at rest in the DRF. For a given flat RSE string shape specified by a 2-d curve $\vec{R}(s)=\left[R_{1}(s), R_{2}(s), R_{3}(s)=0\right]$, this special mass line density $\mu(s)$ is obtained from Eq. (1) with $\partial^{2} \vec{R} / \partial t^{2}=0$ and $\vec{f}_{\text {ext }}$ as in Eq. (6). The resulting differential equations for $T(s)$ and $\mu(s)$ can be integrated exactly, yielding the magical mass line density $\mu(s)$,

$$
\ln \left[\frac{\mu(s)}{\mu(0)}\right]=-\int_{0}^{s} d s^{\prime} \frac{\left(\vec{a}_{n e t}\left(s^{\prime}\right) \cdot \hat{t}\left(s^{\prime}\right)\right)}{K\left(s^{\prime}\right)}+\ln \left[\frac{K(s=0)}{K(s)}\right],
$$

(Golubovic \& Knudsen, 2009). Here,

$$
\vec{a}_{n e t}=-\frac{\partial \Phi{ }_{e f f}}{\partial \vec{R}}
$$

is evaluated at the $\operatorname{RSE}$ point $\left[R_{1}(s), R_{2}(s)\right]$, whereas

$$
K(s)=-\frac{\left(\vec{a}_{n e t}(s) \cdot \hat{n}(s)\right)}{C(s)}=\frac{T(s)}{\mu(s)} .
$$

In Eq. (8'), $C(s)$ is the local RSE string curvature; $C(s)=d \theta / d s$ with $\theta(s)$, the angle between the tangent unit vector $\hat{t}(s)=\partial \vec{R} / \partial s$ and the $R_{1}$ - axis in Figs. 1(a) and (b). The unit vector $\hat{n}(s)$ makes the angle $\theta(s)+\pi / 2$ with the $R_{1}-$ axis. 

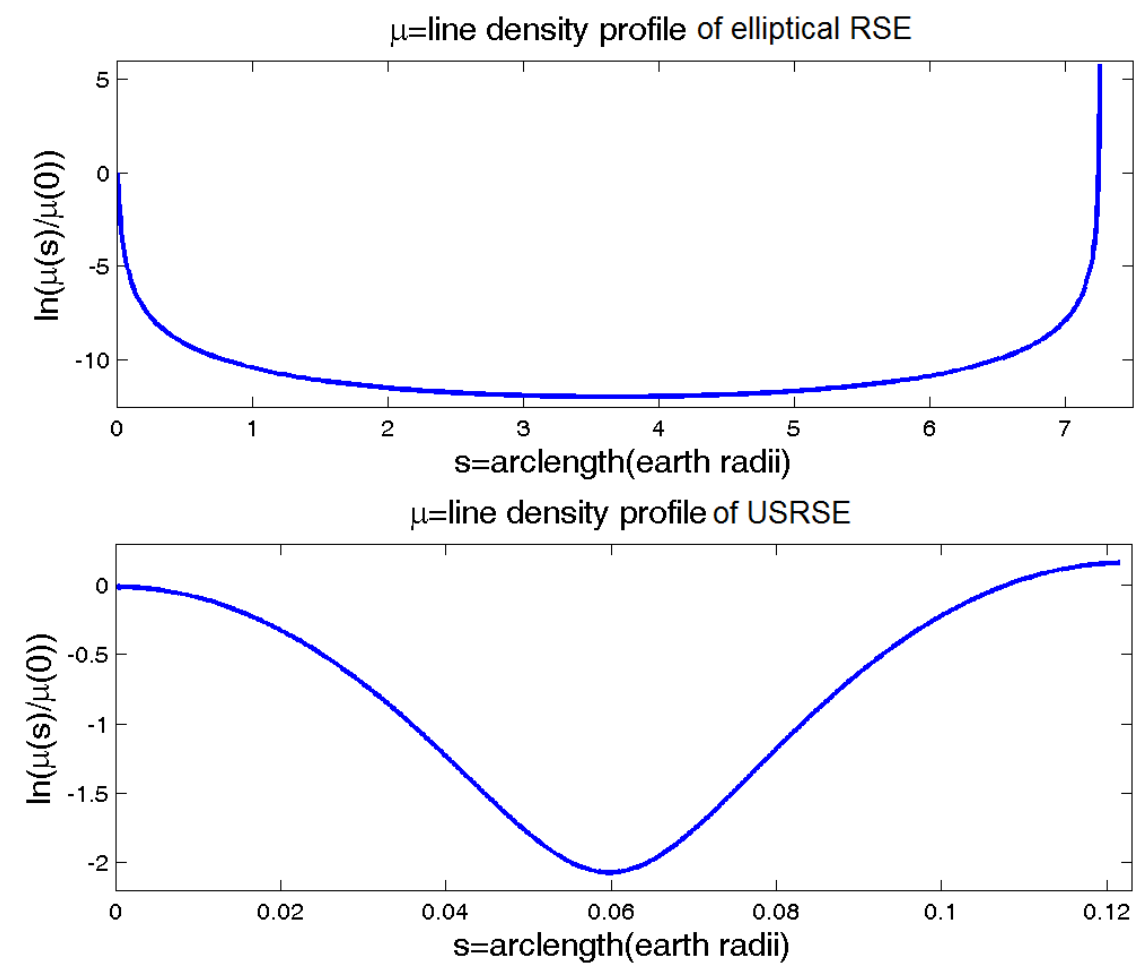

Figure 3. The upper panel: the magical mass distribution [i.e., line density obtained by Eq. (8)] of the RSE with the shape in Figure 1(a) and $T_{R S E}=10.83 \mathrm{~min}$. The lower panel: the magical mass distribution (line density) of the RSE with the shape in Figure 1(b) and $T_{R S E}=4.22 \mathrm{~min}$. Adapted from Golubovic and Knudsen (2009)

The magical mass distributions obtained by applying Eq. (8) to the (initial) RSE shapes in Figure 1, which are used in the simulations discussed in this paper, are shown in Figure 3. The simulations of Knudsen and Golubovic (2014), which are free of the approximation Eq. (6) employed in Eq. (8), indeed show (under the conditions discussed in Sec. 5 in the following) a remarkable stability of the RSE sizes and orientation provided by the magical mass distribution in Eq. (8). We note that $\mu(s)=\rho A(s)$, with $\rho$ the density of the RSE material and $A(s)=$ the string cross-sectional area (that can be made to vary along the RSE by tapered cable design). Thus, by Eq. (8'), the tensile stress obeys the relation,

$$
p(s)=\frac{T(s)}{A(s)}=\rho K(s)
$$

The RSE displayed in Figure 1(b) is a uniform stress RSE (USRSE) for which the tensile stress $p(s)$ is s-independent. For an USRSE, by Eq. (8"), the $K(s)=K=$ const. With this condition, Eq. (8') yields the second order differential equation

$$
\frac{d^{2} R_{2}}{d R_{1}{ }^{2}}=-\frac{1}{K}\left[1+\left(\frac{d R_{2}}{d R_{1}}\right)^{2}\right]\left[a_{2}-a_{1} \frac{d R_{2}}{d R_{1}}\right],
$$

with $a_{i}=-\partial \Phi_{\text {eff }} / \partial R_{i} ; i=1,2$. Differential equation Eq. (8"') can be used to obtain a USRSE shape for any given $K$ and $T_{R S E}$. Figure 1(b) shows thus obtained USRSE shape for $T_{R S E}=4.22 \min \quad$ and $K=\widetilde{K} v_{1}{ }^{2}$. Here, $v_{1}=\left(G M_{\text {earth }} / R_{\text {earth }}\right)^{1 / 2}=7.89 \mathrm{~km} / \mathrm{sec}=1^{\text {st }}$ cosmic speed $\left[R_{\text {earth }}=\right.$ the Earth radius], whereas 
$\widetilde{K}$ is a dimensionless constant. For the USRSE in Figure 1(b), we set $\widetilde{K}=1 / 4$, corresponding, by Eq. (8'), to the USRSE tensile stress $p=\widetilde{K} \rho v_{1}{ }^{2}=20.24 \mathrm{GPa}$ if the USRSE is made of carbon nano-tubes (CNT) with $\rho \approx 1,300 \mathrm{~kg} / \mathrm{m}^{3}$. Thus, pleasingly, the tensile stress $p$ of this USRSE is smaller than the tensile strengths $p_{\max } \approx 60 \mathrm{GPa}$ of single-wall CNT, and $p_{\max } \approx 150 \quad G P a$ of multi-wall CNT (Yu et al., 2000a, Yu et al., 2000b). So, this USRSE is technologically achievable with modern day materials. By Eq. (8) with $K(s)=K=$ const . , and by

$$
-\vec{a}_{n e t} \cdot \hat{t}=\left(\partial \Phi_{e f f} / \partial \vec{R}\right) \cdot(\partial \vec{R} / \partial s)=\partial \Phi_{e f f}(\vec{R}(s)) / \partial s,
$$

we find that the USRSE magical mass line density obeys the equation

$$
\ln \left[\frac{\mu(s)}{\mu\left(s_{1}\right)}\right]=\ln \left[\frac{A(s)}{A\left(s_{1}\right)}\right]=\frac{\Phi_{e f f}(\vec{R}(s))-\Phi_{e f f}\left(\vec{R}\left(s_{1}\right)\right)}{K},
$$

for any $\left(s, s_{1}\right)$. It is depicted in lower panel of Figure 3 for the USRSE in Figure 1(b) [with $T_{R S E}=4.22$ min, and $\widetilde{K}=1 / 4$ ], with $s_{1}=0$ corresponding to the USRSE bottom at the Earth. This line density profile can be technologically achieved by using tapered cable having the cross-sectional area $A(s)$ given by our Eq. (8",',). The USRSE in Figure 1(b) is actually attached to a Linear Space Elevator (LSE) which can also be designed to have a uniform stress $<p_{\max }$ (Pearson, 1975). The LSE line mass density has a discontinuity at the junction between the USRSE and the LSE (to balance the USRSE tension force pulling down the LSE along the $R_{1}$-axis). Away from the junction, the uniform stress LSE line mass density obeys Eq. (8"'") with $\vec{R}(s)=\left(R_{1}=s, R_{2}=0, R_{3}=0\right) \quad$ (Pearson, 1975). We note that unlike the technologically achievable USRSE in Figure 1(b) with $p<p_{\max }$ (for CNT), the Earth based elliptic RSE in Figure 1(a) has a non-uniform stress that actually exceeds the CNT tensile strength in the midsection of the RSE. However, elliptic RSEs built on dwarf planets such as asteroids Ceres and Vesta would have a tensile stress $p<p_{\max }$ (for CNT). They are thus technologically achievable.

Periodic like motion of sliding climbers (seen in the simulations in Figure 2) goes on along nearly constant shape RSE strings. This climber motion can be understood by means of Eq. (6). For a time-independent RSE shape $\vec{R}(s)$, the Eq. (1'), in combination with Eq. (6), reduces to

$$
\ddot{s}=-\hat{t}(s) \cdot \frac{\partial \Phi_{e f f}}{\partial \vec{R}(s)}=-\frac{\partial \vec{R}(s)}{\partial s} \cdot \frac{\partial \Phi_{e f f}}{\partial \vec{R}(s)}=-\frac{\partial \Phi_{e f f}(\vec{R}(s))}{\partial s},
$$

yielding the conservation law

$$
\frac{1}{2}\left(\frac{d s(t)}{d t}\right)^{2}+\Phi_{e f f}[\vec{R}(s(t))]=\text { const }
$$

Eq. (9) is isomorphic to the familiar conservation law describing oscillatory 1-d motion of a particle with the position $s(t)$ in the potential $U(s)=\Phi_{e f f}(\vec{R}(s))$. Strikingly, in this potential, sliding climbers simply oscillate between two turning points, one of which is close to the Earth (starting point) whereas the other one is in outer space; see Figs. 1 and 2. In fact, the RSE bottom (the point $s=0$ ) becomes a local maximum of the potential $U(s)$ (seen by sliding climbers) provided the RSE angular frequency $\Omega_{\text {RSE }}$ is bigger than the minimal frequency,

$$
\Omega_{\min } \cong \frac{v_{1}}{R_{\text {earth }}}\left(1+R_{\text {earth }}|C(s=0)|\right)^{1 / 2}=\frac{v_{1}}{R_{\text {earth }}}\left(1+\frac{1}{\widetilde{K}(s=0)}\right)^{1 / 2},
$$

with $\widetilde{K}(s)=K(s) / v_{1}{ }^{2}$ (Golubovic \& Knudsen, 2009; Knudsen \& Golubovic, 2014). Due to this, for $\Omega_{R S E}>\Omega_{\min }$, i.e., $T_{R S E}<T_{\max }=2 \pi / \Omega_{\min }$, a climber initially at rest will start moving up no matter how close is its initial position to the RSE bottom $\left[R_{1}=R_{\text {earth }}, R_{2}=R_{3}=0\right.$ )] in Figs. 1(a) and (b), at $s=0$. For 
the elliptical RSE in Figure 1(a) with the semi-axes $b=0.5 R_{\text {earth }}, a=3.2107 R_{\text {earth }}$, the climbing threshold RSE period $T_{\max }=22.71 \mathrm{~min}$. This is bigger than the $T_{\text {RSE }}$ of $10.83 \mathrm{~min}$ of the elliptic RSE in Figure 1(a), yielding the oscillatory sliding climber dynamics seen in the simulations in Figure 2, upper panel. For an USRSE with $\widetilde{K}(s)=1 / 4$, by Eq. (10), $T_{\max }=37.78 \mathrm{~min}$. This is bigger than the $T_{R S E}$ of $4.22 \mathrm{~min}$ of the USRSE in Figure 1(b), yielding the oscillatory sliding climber dynamics seen in the simulations in Figure 2, lower panel. We note that the USRSE point having the maximum distance $R_{2}$ away from the $R_{1}$ - axis in Figure 1(b) has the speed $\Omega_{R S E} \cdot\left(R_{2}\right)_{\max } \cong v_{1}=1^{\text {st }}$ cosmic speed (for the USRSE with $T_{R S E}=4.22 \mathrm{~min}$ ). Thus, the USRSE loop in Figure 1(b) can be used for launching satellites. We will discuss potential applications of the RSE in Sec. 6. It is significant to note that [by using differential Eq. $\left(8^{\prime \prime \prime}\right)$ ] the USRSE loops can be designed with their bottoms anywhere above the Earth surface (e.g., above the dense atmospheric layer, to avoid air-resistance).

\section{Shape Stability of RSE and Crumpling Transition}

Numerical simulations of RSEs reveal an interesting morphological phase transition of the RSE strings that occurs with changing the (initial) RSE angular frequency $\Omega_{\text {RSE }}$, i.e., its period $T_{R S E}$ (Golubovic \& Knudsen, 2009; Knudsen \& Golubovic, 2014). This transition was seen both in the USRSE and the elliptical RSE. E.g., for the elliptical RSE in Fig 1(a), it occurs at a critical value for the RSE period $T_{\text {crit }} \approx 17 \mathrm{~min}$. For $T_{R S E}<T_{\text {crit }}$, the tension field $T(s, t)$ remains everywhere positive. It exhibits only small oscillations around $T(s, t=0)>0$ given by Eqs. (8) and ( $\left.8^{\prime}\right)$. However, for $T_{R S E}>T_{c r i t}$, the RSE string (in both the elliptical RSE and the USRSE) undergoes a dramatic shape change and chaotic long time dynamics: Tension field develops a noise like behavior in which $T(s, t)$ assumes both positive and negative values. In effect, the RSE string crumples due to the buckling of the string sections that are under locally negative $T(s, t)$. Macroscopically, the string crumpling triggers a narrowing of the RSE initial shape, displayed in Figure 4(d) for the elliptical RSE with $T_{R S E}=21.66 \mathrm{~min}$. The narrowing eventually turns initially elliptic RSE into two nearly independently fluctuating linear type space elevators connecting the massive elevator top and bottom regions. Chaotic dynamics of the two elevator's branches reflects an ergodic-like (thermal equilibrium like) string state similar to that of the directed polymers (Kardar, 2007) stretched between the RSE top and bottom. Related to the RSE narrowing is the dynamics of the RSE angular momentum $L_{1}$ about the $R_{1}$-axis (in the frame rotating with the Earth as in Figure 1); see Figure 4(c) for the elliptical RSE with $T_{R S E}=21.66 \mathrm{~min}:$ The $L_{1}$ decays to zero over a two week period. [In contrast to this, for $T_{R S E}>T_{\text {crit }}$, the USRSE narrows and loses its $L_{1}$ only partially.] As seen in Figs. 4 (a) and (b) at $T_{R S E}=21.66 \mathrm{~min}$, these phenomena destabilize the position of the elliptical RSE top. It drifts away from its initial position (at 0.8 Earth radii above geostationary level) to a new slightly higher position around which the RSE top continues to chaotically oscillate.

In drastic contrast to this, for $T_{\text {RSE }}<T_{\text {crit }}$, the string shapes of both the elliptic RSE and the USRSE remain nearly the same as in their initial configuration in Figs. 1(a) and (b), i.e., no RSE narrowing occurs. Related to this, as evidenced in Figure 4(c) for the elliptical RSE with $T_{R S E}=10.83 \mathrm{~min}$, the RSE angular momentum $L_{1}$ is nearly constant in time, whereas the RSE top exhibits only very small oscillations around its initial position; see Figure 4(a) and (b). 
a)

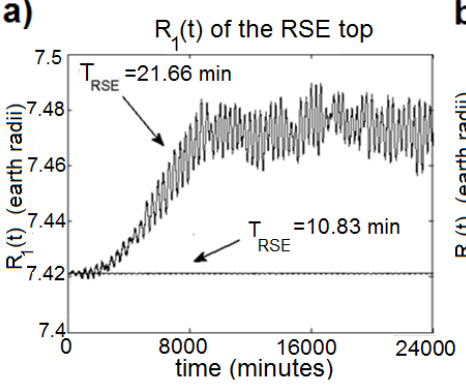

b)
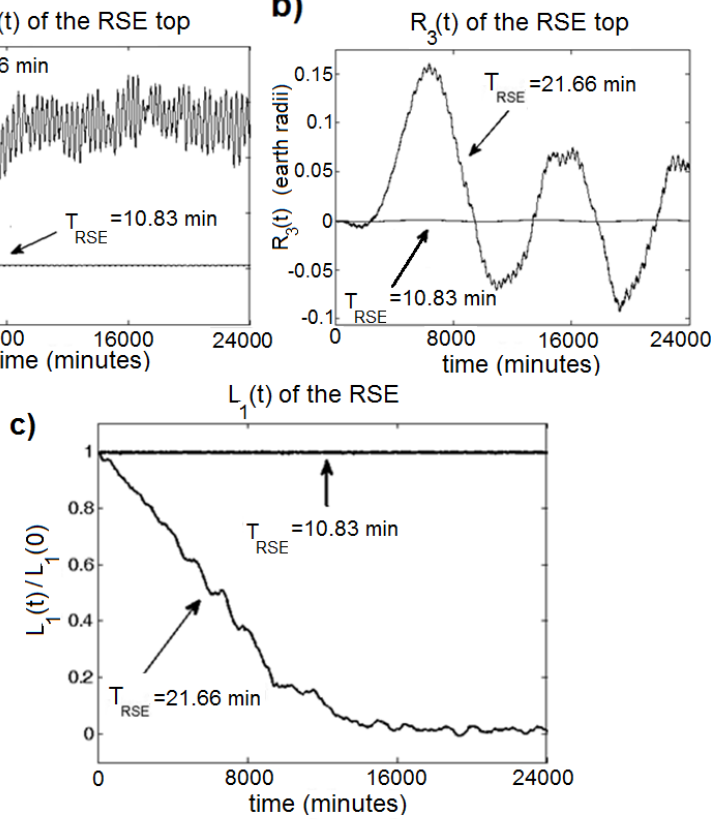

d)

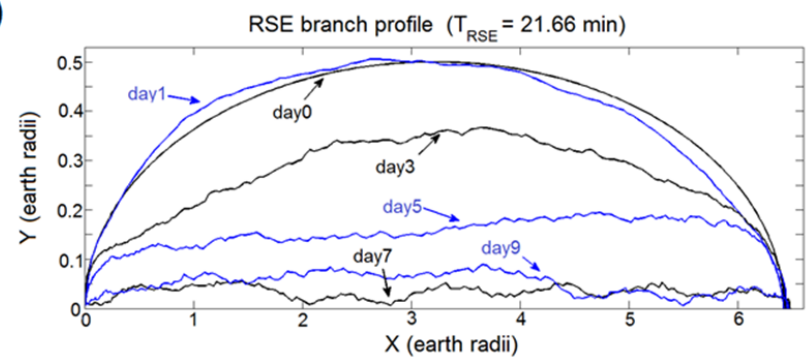

Figure 4. From the simulations of Knudsen and Golubovic (2014): For the elliptic RSE in Figure 1(a), the RSE top coordinates $R_{1}(t)$ in (a), $R_{3}(t)$ in (b), and, in (c), the evolution of the RSE angular momentum $L_{1}$ about the $R_{1}$-axis (in the frame rotating with the Earth), for $T_{R S E}=10.83 \mathrm{~min}$ and $T_{R S E}=21.66 \mathrm{~min}$. In (d), the evolution of the RSE profile (of one of its two branches), for $T_{R S E}=21.66 \mathrm{~min}$ over the first ten days. Here, for any RSE point $\mathrm{P}$, the $Y$ is its distance away from the (instantaneous) axis $A$ connecting the RSE bottom and top, and $X$ is the distance between the normal projection of $\mathrm{P}$ onto the axis $A$ and the RSE bottom point (at

$$
X=0)
$$

\section{Using RSEs to Launch Spaceships and Satellites}

By the discussions of Sec. 4, RSE is a rapid extraterrestrial transportation system which requires no internal engines for the climbers sliding along the elevator strings. Climbers motion is naturally facilitated by employing basic natural phenomena, the inertial forces due to the internal RSE rotation around the $\mathrm{R}_{1}$-axis in Figure 1 . As noted before in Sec. 4, RSEs can be used to launch satellites and interplanetary spaceships (Knudsen \& Golubovic, 2014). In this section we will discuss this RSE capability in more detail. It is also significant to note that the RSE strings can be used to host space stations and research posts. Sliding climbers can be then used to transfer useful loads and humans from the Earth to these extraterrestrial locations.

Satellites and spaceships transported by sliding climbers can be released (launched) along RSEs. Let us look at a climber that has started its motion at near rest at the RSE tying position with the Earth in Figure 1 [there, $\left.|\vec{R}(s=0)|=R_{\text {earth }}\right]$. Let us then look at an object released from the climber when it reaches the RSE position $\vec{R}(s)=\left[R_{1}(s), R_{2}(s), R_{3}(s)=0\right]$ in the DRF. The released object's speed in the DRF, that is its speed along the tangent at $\vec{R}(s)$ is $d s / d t$. This tangential velocity is obtained by Eq. (9) yielding,

$$
\frac{1}{2}\left(\frac{d s(t)}{d t}\right)^{2}=\Phi_{e f f}[\vec{R}(s(0)=0)]-\Phi_{e f f}[\vec{R}(s(t))] .
$$

Using here the Eqs. (5) and (7), we find, 


$$
\frac{1}{2}\left(\frac{d s(t)}{d t}\right)^{2}=-G \frac{M_{\text {earth }}}{R_{\text {earth }}}+G \frac{M_{\text {earth }}}{|\vec{R}(s)|}+\frac{1}{2} \Omega_{\text {earth }}^{2}\left[R_{1}(s)\right]^{2}+\frac{1}{2}\left(\Omega_{R S E}^{2}+\frac{1}{2} \Omega_{\text {earth }}^{2}\right)\left[R_{2}(s)\right]^{2} .
$$

Of the practical interest is the released object speed observed in the inertial frame. Consider, for example, the situation in which the object is released when the elevator loop is in the plane of Figure 1(a, upper panel) or Figure1 (b) (then the $\mathrm{R}_{2}$ axis of DRF points along the north-south direction). Let the object be released from a climber which is on the lower branch of RSE in Figure 1. The RSE velocity at this point is in the direction of the Earth rotation and has the magnitude

$$
v_{R S E}=\Omega_{R S E}\left|R_{2}(s)\right|+\Omega_{\text {earth }} R_{1}(s),
$$

in the inertial frame. This velocity points into the plane of Figure 1. In addition to this velocity, the released object also has the tangential velocity $d s / d t$ which is in the plane of Figure 1 . Thus, by Pythagorean Theorem, the total released object speed in the inertial frame, $v_{\text {released }}$ satisfies

$$
v_{\text {released }}=\sqrt{\left(v_{R S E}\right)^{2}+(d s / d t)^{2}} .
$$

By Eqs. (12) through (14), the speed of the released object in the inertial frame satisfies the equation

$$
\begin{aligned}
& \frac{1}{2}\left(v_{\text {released }}\right)^{2} \\
& =-G \frac{M_{\text {earth }}}{R_{\text {earth }}}+G \frac{M_{\text {earth }}}{|\vec{R}(s)|}+\left(\Omega_{R S E}^{2}+\frac{1}{4} \Omega_{\text {earth }}^{2}\right)\left[R_{2}(s)\right]^{2} \\
& +\Omega_{R S E} \Omega_{\text {earth }}\left|R_{2}(s)\right| R_{1}(s)+\Omega_{\text {earth }}^{2}\left[R_{1}(s)\right]^{2},
\end{aligned}
$$

if the object is released when the elevator loop is in the plane of Figure 1(a, upper panel) or Figure 1 (b), from a climber which is on the lower branch of RSE in Figure 1. If this speed is large enough, the released object will unbind from the Earth, and approach infinity with the speed $v_{\infty}$ ("escaping speed") that can be obtained from the mechanical energy conservation law,

$$
\frac{1}{2}\left(v_{\infty}\right)^{2}=\frac{1}{2}\left(v_{\text {released }}\right)^{2}-G \frac{M_{\text {earth }}}{|\vec{R}(s)|} .
$$

By Eqs. (15) and (16),

$$
\begin{aligned}
& \frac{1}{2}\left(v_{\infty}\right)^{2} \\
& =-\left(v_{1}\right)^{2}+\left[\Omega_{R S E} R_{2}(s)\right]^{2}\left[1+\left(\frac{\Omega_{\text {earth }}}{2 \Omega_{\text {RSE }}}\right)^{2}+\frac{\Omega_{\text {earth }} R_{1}(s)}{\Omega_{\text {RSE }} R_{2}(s)}+\left(\frac{\Omega_{\text {earth }} R_{1}(s)}{\Omega_{\text {RSE }} R_{2}(s)}\right)^{2}\right] .
\end{aligned}
$$

Above, we introduced the first cosmic speed $v_{1}=\left(G M_{\text {earth }} / R_{\text {earth }}\right)^{1 / 2}=7.89 \mathrm{~km} / \mathrm{sec}$. Again we stress that the Eq. (17) applies if the object is released when the elevator loop is in the plane of our Figure 1(a, upper panel) of Figure 1(b), from a climber which is on the lower branch of RSE in Figure 1. This particular case is significant because the enhancement of the released object speed provided by the rotation of the Earth is at its maximum (for the RSE rotating around the $\mathrm{R}_{1}$ axis in the direction indicated in Figure 1). For the interesting (for RSE systems) situations with $\Omega_{R S E}>>\Omega_{\text {earth }}$ [see the discussions following Eq. (5)], the results in Eqs. (13), (15) and (17) reduce to approximate yet more illuminating results,

$$
\begin{gathered}
v_{R S E} \approx \Omega_{R S E}\left|R_{2}(s)\right|, \\
\frac{1}{2}\left(v_{\text {released }}\right)^{2} \approx-G \frac{M_{\text {earth }}}{R_{\text {earth }}}+G \frac{M_{\text {earth }}}{|\vec{R}(s)|}+\Omega_{R S E}^{2}\left[R_{2}(s)\right]^{2},
\end{gathered}
$$

while the escaping speed ("speed at infinity") approximately satisfies the simple equation,

$$
\frac{1}{2}\left(v_{\infty}\right)^{2} \approx-\left(v_{1}\right)^{2}+\left[\Omega_{R S E} R_{2}(s)\right]^{2} .
$$

The approximate results $\left(13^{\prime}\right),\left(15^{\prime}\right)$, and (17') are equivalent to ignoring the Earth rotation (setting $\left.\Omega_{\text {earth }}=0\right)$ in the exact results in Eqs. (13), (15), and (17). Thus, the above approximate results are significant also because 
they (approximately) apply to the objects released from the RSE at any orientation of the rotating RSE plane relative the $\mathrm{R}_{1}-\mathrm{R}_{2}$ plane in Figure 1. [We stress that $\left|R_{2}(s)\right|$ in the above equations is the distance between the release point and the $\mathrm{R}_{1}$ axis.]

The approximate results (13'), (15'), and (17') offer a better insight into the launching actions of the RSE. By Eq. (17'), the released object (spaceship) will escape to an interplanetary travel $\left[\left(v_{\infty}\right)^{2}>0\right]$ if the RSE speed of the release point of $\left(=\Omega_{R S E}\left|R_{2}(s)\right|\right)$ is bigger than the first cosmic speed $v_{l}$. By the Eq. (17'), the highest possible escaping speed $v_{\infty}$ is achieved if the object is released from a sliding climber at the RSE point with the maximum value of $\left|\mathrm{R}_{2}\right|$. For example, for the elliptic RSE in the figure 1(a), this point is the midpoint of the RSE, with $\left|R_{2}\right|_{\max }=b=0.5 R_{\text {earth }}$ at $R_{1}=a+R_{\text {earth }}=4.2107 R_{\text {earth. At }}$ this point, with $T_{R S E}=10.83$ min, one has $\Omega_{R S E}\left|R_{2}(s)\right|=3.9 v_{1}$. With this value, the Eq. (17') predicts the value of the highest possible escaping speed (speed at infinity) from this RSE to be $\left(v_{\infty}\right)_{\max } \approx 5.3310 v_{1}$ which is only slightly smaller than $v_{\infty}=5.5200 v_{1}$ as obtained by using the exact Eq. (17). We note that for the marginal case with $\Omega_{R S E}\left|R_{2}(s)\right|=v_{1}$, whence the approximation Eq. (17') predicts $v_{\infty}=0$, the exact equation (17) yields an $\left(v_{\infty}\right)^{2}>0$, meaning that the object still unbinds with a small escape velocity at infinity. A situation like this is (incidentally) realized in the USRSE in the Figure 1(b), with $T_{R S E}=4.22 \mathrm{~min}$. For its point with the maximum value of $\left|\mathrm{R}_{2}\right|\left(\left|R_{2}\right|_{\max }=0.052 R_{\text {earth }}\right.$ at $R_{1}$ $=1.02 R_{\text {earth }}$ ) we find $\Omega_{R S E}\left|R_{2}(s)\right|=1.04 v_{l}$ (which is only slightly above $v_{l}$ ). The approximate Eq. (17') would then yield $v_{\infty} \approx 0.4040 v_{1}$, whereas the exact Eq. (17) gives $v_{\infty}=0.54303 v_{1}$ for this case [if the object is released when the elevator loop is in the plane of our Figure 1, from a climber which is on the lower branch of USRSE in Figure 1(b)].

The shape of a USRSE loop is determined by solving the differential equation ( 8 ','), and thus it depends on the value of $T_{R S E}=2 \pi / \Omega_{R S E}$. In Figure 5 (upper panel), we display the USRSE shapes for several different values of $T_{R S E}$, all for the same value of the parameter $\widetilde{K}=1 / 4$ (corresponding to the string tensile stress $p=20.24 \mathrm{GPa}$ if the USRSE is made of carbon nano-tubes, see Sec. 4). In Figure 5 (lower panel), we plot, versus $T_{R S E}$, the USRSE speed $\Omega_{R S E}\left|R_{2}(s)\right|_{\max } \quad$ as well as the speed at infinity $v_{\infty}$ of an object released from a climber at $\left|R_{2}(s)\right|_{\max }$ on the lower branch of USRSE in Figure 1(b) when this branch is in the plane of our Figure 1. With a known RSE shape, this speed can be calculated from Eq. (17). Note that $v_{\infty}$ vanishes at a characteristic value of $T_{R S E}$ of about $6 \mathrm{~min}$.
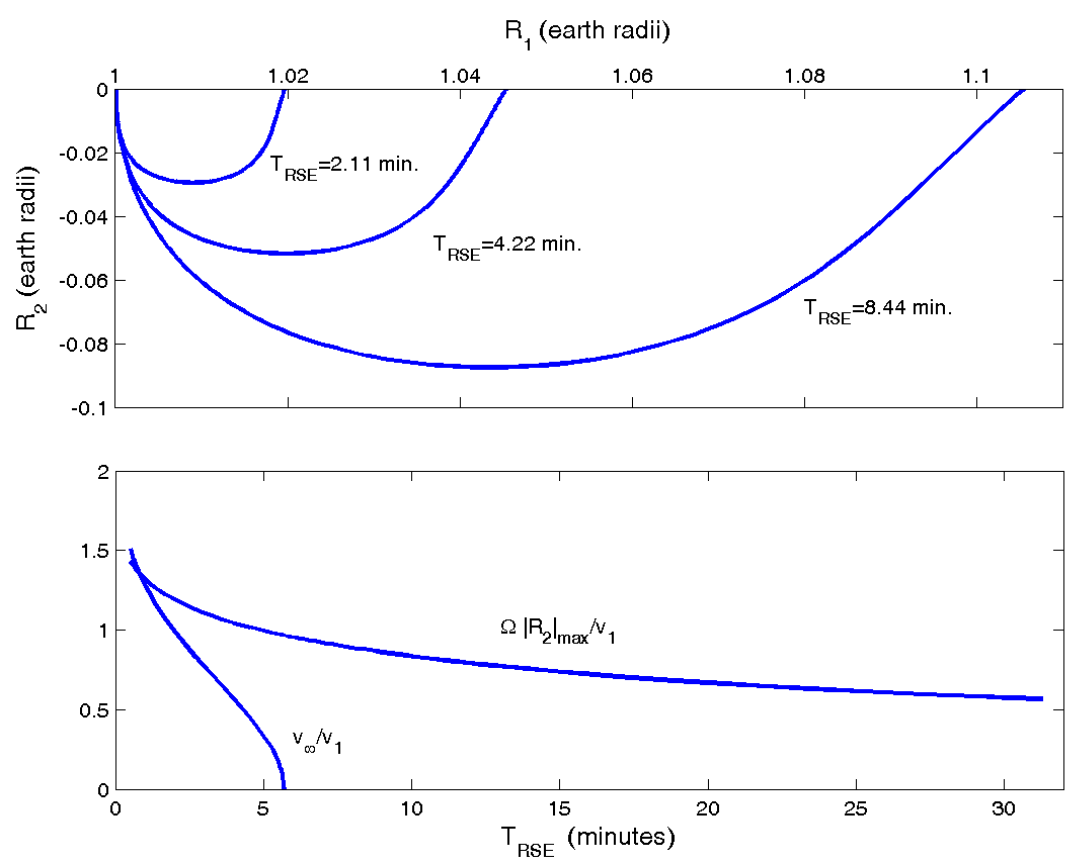

Figure 5. In the upper panel, we plot the USRSE loop shapes obtained for several different values of $T_{R S E}$, all for the same value of the parameter $\widetilde{K}=1 / 4$. In the lower panel, we plot, versus $T_{R S E}$, the USRSE speed $\Omega_{R S E}\left|R_{2}(s)\right|_{\max }$ as well as the speed at infinity $v_{\infty}$ of an object released from a sliding climber at $\left|R_{2}(s)\right| \max$ on the lower branch of USRSE in Figure 1(b) when this branch is in the plane of our Figure 1. It is obtained from Eq. (17). The two speeds are given in units of the first cosmic speed $v_{1}$. Adapted from Knudsen and Golubovic (2014) 
Thus far we have discussed the case with no sliding friction between the climber and the RSE string. Only then Eq. (9) applies. The friction may be significantly depressed, e.g., by magnetic levitation. With some friction present, climbers would eventually stop near the RSE point minimizing the effective potential seen by the climber in DRF, $U(s)=\Phi_{\text {eff }}(\vec{R}(s))$. From the equipotentials displayed in Figure 1, this point occurs very close to the RSE point maximizing its $R_{2}$ coordinate in Figure 1. Interestingly, as noted above, the USRSE point having the maximum distance $\left|R_{2}\right|$ away from the $R_{1}$ - axis in Figure 1(b) has the speed $\Omega_{R S E} \cdot\left|R_{2}\right|_{\max } \cong v_{1}=1^{\text {st }}$ cosmic speed (for the particular USRSE shown in Figure 1(b), with $T_{R S E}=4.22$ min and $\widetilde{K}=1 / 4$; see Sec. 4) Thus, the USRSE loop in Figure 1(b) can be used for launching satellites carried from the Earth by a sliding climber. Indeed, due to the friction, the climber would eventually stop close to the USRSE point having the maximum distance away from the $R_{1}$-axis in Figure 1(b). At this point, the stopped climber rotates with the RSE with the speed $\cong v_{1}=1^{\text {st }}$ cosmic speed. Thus, the climber can directly release the carried satellite into a nearly circular low Earth orbit.

\section{Physics of Untied RSE}

What will happen if one unties the elliptic RSE (ERSE) in Figure 1 from the Earth? This interesting question is investigated by Knudsen and Golubovic (2015). Interestingly, it was found that the tying may not be needed at all to achieve the stable double rotating motion of ERSE. In fact, the magical mass distribution $\mu(s)$ in Eq. (8) does not assume that the loopy ERSE is tied. Thus, it is in principle possible that an untied ERSE exhibits persistent shape and everlasting double rotating motion much like the tied ERSE. This intriguing possibility was explored by studying the dynamics of the untied elliptical RSE (Knudsen \& Golubovic, 2015). The actual untied ERSE behavior was found to depend on the length of its long semi-axis $a$ (along the $\mathrm{R}_{1}$-axis in Figure 1). The study shows that there are two characteristic values of $a$, called $a_{\text {hopping }}$ and $a_{\text {unbinding. If }} a<a_{\text {hopping, }}$, the untied ERSE exhibits nearly the same dynamics as a tied ERSE. That is, its bottom and top points execute only very

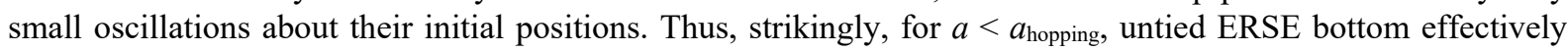
remains quasi-tied to the Earth. It remains close to the Earth as if the ERSE were tied to the Earth. On the other side, if $a>a_{\text {hopping, }}$, the untied ERSE as a whole hops away and then it falls back to the Earth. The amplitude of this hopping (maximum height reached by the RSE bottom) increases with increasing $a$ and it diverges as $a$

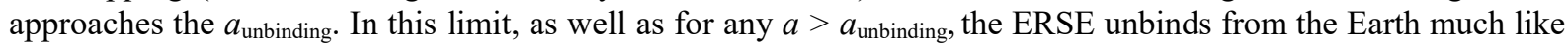
an object with a speed above the second cosmic speed.

\section{Summary}

In summary, the RSEs are rapid outer space transportation systems that require no internal engines for the climbers sliding along the elevator strings. RSE strings exhibit interesting nonlinear dynamics and statistical physics phenomena. RSEs' action fundamentally employs truly basic natural phenomena -- gravitation and inertial forces. Satellites and space-crafts carried by sliding climbers can be released (launched) along RSEs. RSE strings can host space stations and research posts. Sliding climbers can be then used to transport useful loads and humans from the Earth to these outer space locations. The RSE exhibits a variety of interesting dynamical phenomena explored by numerical simulations. Thanks to its special design aided by its magical mass distribution, the RSE exhibits persistent shape and enduring double rotating motion. Under some conditions however the RSE may undergo a morphological transition to a chaotic state reminiscent of fluctuating directed polymers encountered in the statistical physics of strings and membranes.

\section{Acknowledgments}

The authors thank WVHTC and NASA for the sub-grant WVHTC-W-NASA-IR-07-1367 of the Grant NNX06AE557: Innovative Research for Next Generation Space Exploration.

\section{References}

Artsutanov, Y. V. (1960, July 31). V Kosmos na Elektrovoze (To the Cosmos by Electric Train). Komsomolskaya Pravda (in Russian), front page of Sunday Supplement. English translation retrieved from http://www.spaceward.org/documents/Artsutanov_Pravda_SE.pdf

Clarke, A. C. (1978). The Fountains of Paradise. New York, NY: Harcourt Brace Jovanovich.

Clarke, A. C. (1981). The Space Elevator: 'Thought Experiment' or Key to the Universe?. Advances in Earth Oriented Applied Space Technology Vol. 1, 39-48. London, UK: Pergamon Press. Retrieved from http://www.islandone.org/LEOBiblio/CLARK1.HTM

Edwards, B. C., \& Westling, E. A. (2003). The Space Elevator: A Revolutionary Earth-to-Space Transportation System. USA: BC Edwards. 
Fitzgibbons, T. C., Guthrie, M., En-shi Xu E., Crespi, V. H., Davidowski, S. K., ... Badding, J. V. (2014), Benzene-derived carbon nanothreads, Nature Materials, 14, 43-47. http://dx.doi.org/10.1038/nmat4088

Golubović, L., \& Knudsen, S. (2009). Classical and statistical mechanics of celestial scale spinning strings: Rotating space elevators. Europhysics Letters, 86(3), 34001. http://dx.doi.org/10.1209/0295-5075/ $86 / 34001$

Kardar, M. (2007). Statistical Physics of Fields. Cambridge, UK: Cambridge University Press.

Knudsen, S., \& Golubović, L. (2014). Rotating space elevators: Physics of celestial scale spinning strings. European Physical Journal Plus, 129, 242. http://dx.doi.org/10.1140/epjp/i2014-14242-8

Knudsen, S., \& Golubović, L. (2015). Physics of untied rotating space elevators. European Physical Journal Plus, 130, 243. http://dx.doi.org/10.1140/epjp/i2015-15243-9

Landau, L. D., \& Lifshitz, E. M. (1976). Mechanics, Course in Theoretical Physics, Vol. 1. London, UK: Pergamon Press.

Lvov, V. (1967). Sky-Hook: old idea. Science, 158, 946-947. http://dx.doi.org/10.1126/science.158.3803.946

Nelson, D. R. (2002). Defects and Geometry in Condensed Matter Physics. Cambridge, UK: Cambridge University Press.

Nelson, D. R., Piran, T., \& Weinberg, S. (Eds.). (1988). Statistical Mechanics of Membranes and Surfaces. Singapore: World Scientific.

Pearson, J. (1975). The Orbital Tower: A Spacecraft Launcher Using the Earth's Rotational Energy. Acta Astronautica, 2, 785-799. http://dx.doi.org/10.1016/0094-5765(75)90021-1

Yu, M. F., Files, B. S., Arepalli, S., \& Ruoff, R. S. (2000a). Tensile Loading of Ropes of Single Wall Carbon Nanotubes and their Mechanical Properties. Physical Review Letters, 84, 5552-5555; http://dx.doi.org/10. 1103/PhysRevLett.84.5552

Yu, M. F., Lourie O., Dyer M. J., Moloni, K., Kelly, T. F., \& Ruoff. R. S. (2000b). Strength and breaking mechanism of multiwalled carbon nanotubes under tensile load, Science 287, 637-640. http://dx.doi.org/10. $1126 /$ science.287.5453.637

\section{Copyrights}

Copyright for this article is retained by the author(s), with first publication rights granted to the journal.

This is an open-access article distributed under the terms and conditions of the Creative Commons Attribution license (http://creativecommons.org/licenses/by/4.0/). 